\title{
Eye fluke infection and lens size reduction in fish: a quantitative analysis
}

\author{
Anssi Karvonen ${ }^{1, *}$, Otto Seppälä ${ }^{1,2}$ \\ ${ }^{1}$ Department of Biological and Environmental Science, University of Jyväskylä, PO Box 35, 40014 Jyväskylä, Finland \\ ${ }^{2}$ Present address: EAWAG, Department of Aquatic Ecology and ETH-Zürich, Institute of Integrative Biology, \\ Überlandstrasse 133, PO Box 611, 8600 Dübendorf, Switzerland
}

\begin{abstract}
Parasites have a variety of harmful effects on their hosts, some of which may be overlapping or complementary and thus easily overlooked but which are still important for the overall severity of infection. We investigated the effect of Diplostomum sp. eye fluke infection on the size of the eye lens in a range of wild and farmed fish species and those exposed to controlled parasite infection. We found that asymmetry in intensity of infection between the right and left lens of an individual fish affected lens size such that the lens with the higher intensity of infection was smaller. Interestingly, however, this was observed only in 3 of the 10 species studied (whitefish, smelt and sea trout) although in these 3 species the effect had already became evident at low intensities of infection. Furthermore, the average lens size was significantly smaller in experimentally exposed whitefish Coregonus lavaretus with a higher intensity of infection than the controls, emphasising the sensitivity of this species to parasite-induced changes in lens size. Reduction in lens size may contribute to the deleterious effects of cataract formation by intensifying the effects of individual parasites in the lens. It may also directly affect the overall optical performance of the lens and already impair host vision at low intensities of infection.
\end{abstract}

KEY WORDS: Parasite infection · Trematoda $\cdot$ Lens diameter · Optical performance $\cdot$ Parasiteinduced cataracts · Diplostomiasis · Fish farming $\cdot$ Coregonus lavaretus

Resale or republication not permitted without written consent of the publisher

\section{INTRODUCTION}

Quantification of the negative effects of parasites on their hosts is one of the key features of epidemiology and medical parasitology (Hudson et al. 2002). From an applied point of view, this is particularly important with disease agents affecting the human population, and also with those affecting the health and productivity of our livestock. In general, parasites may affect the host by causing physiological injuries or, more indirectly, by affecting host condition and the subsequent level of reproduction and survival. Some of these effects, however, may be overlapping or complementary and thus easily overlooked, but they are nevertheless important when estimating the overall harmfulness of infection.
Eye flukes of the genus Diplostomum are ubiquitous parasites of freshwater fish and complete their life cycle by passing successively through 3 different hosts: an avian definitive host, a snail first intermediate host and a fish second intermediate host. The taxonomy of these parasites is complex and not completely resolved, as several species with close morphological and ecological resemblance have been described. Thus, in the present study, we refer to these parasites as Diplostomum sp. Metacercarial stages of the parasites are found in the eyes of fish, where species infecting lenses (note that some species infect humour; Karvonen et al. 2006a) cause cataracts, a disease known as diplostomiasis. Cataract coverage of the lens area increases with intensity of infection (Karvonen et al. 2004a) and causes deleterious secondary effects in fish 
such as increased susceptibility to predation (Seppälä et al. 2005). The infection is also known to impair feeding and growth of fish (Crowden \& Broom 1980, Owen et al. 1993, Buchmann \& Uldal 1994) although these effects have not been quantitatively investigated in relation to cataracts. The parasites are also found at fish farms (Stables \& Chappell 1986, Field \& Irwin 1994, Karvonen et al. 2006b), where they cause blindness and impaired growth and condition of the fish. In addition to the ocular pathology in terms of cataracts, there is some evidence suggesting that smaller lens size is associated with high-level infection in rainbow trout (Shariff et al. 1980). However, this has neither been quantified in detail nor investigated at the host species level, although it may have important implications for both the optical performance of the lens and the relationship between intensity of infection and cataract coverage.

In this paper, we first examined the effects of Diplostomum sp. infection on the size of eye lenses within host individuals in wild and farmed fish populations. This examination is designed to reveal differences in responses between host species as well as the effects of asymmetrical distribution of parasites at an individual level. Second, to explore the more general effect of infection (variation in intensity of infection between host individuals) on lens size, we subjected one of the fish species studied from wild and farmed environments to experimental exposure with controlled parasite doses.

\section{MATERIALS AND METHODS}

We first examined the relationship between intensity of infection and lens size in wild fish populations. One hundred individuals of each of 7 species (whitefish Coregonus lavaretus, smelt Osmerus eperlanus, bleak Alburnus alburnus, dace Leuciscus leuciscus, roach Rutilus rutilus, ruff Gymnocephalus cernuus, and three-spined stickleback Gasterosteus aculeatus) were caught with fish traps and gill nets from a brackish water area in Kiviniemi $\left(65^{\circ} 06^{\prime} \mathrm{N}, 25^{\circ} 19^{\prime} \mathrm{E}\right)$, Bothnian Bay (Baltic Sea) in May 2006. Fish were killed immediately, put on ice and brought to the laboratory where they were measured for total body length and both lenses were examined for parasites. The diameter of the lenses was also measured under a microscope to the nearest $0.04 \mathrm{~mm}$.

Farmed fish species (3 Finnish populations of Atlantic salmon Salmo salar, 4 populations of sea trout S. trutta m. trutta, 1 population of brown trout $S$. trutta m. lacustris, and 1 population of whitefish Coregonus lavaretus) were sampled from a commercial fish farm in Finland in August 2007. Fifty individuals of each population were haphazardly selected from 2 or 3 different tanks except for brown trout, which came from a single pond. Fish were processed as described above.

Under laboratory conditions, we exposed whitefish Coregonus lavaretus to Diplostomum sp. cercariae. A total of 1500 whitefish fry (mean weight less than $1 \mathrm{~g}$ ) were obtained from a commercial fish farm. Fish were allowed to grow in a $1200 \mathrm{l}$ tank on an ad libitum diet for $10 \mathrm{wk}$, after which they had reached a mean length and weight of $121.6 \pm 1.3 \mathrm{~mm}(\mathrm{SE})$ and $13.2 \pm 0.5 \mathrm{~g}$, respectively. During this time and previous maintenance at the fish farm, the fish acquired a low-level Diplostomum sp. infection (mean $=6.7 \pm 0.2$ parasites fish $^{-1}$ ) from the incoming water. However, no further exposure occurred after this period in the laboratory, as parasite transmission was inhibited by a natural decrease in water temperature in the basin of the nearby lake where the water was obtained (see also Karvonen et al. 2004b). Fish, however, were maintained at a constant teperature $\left(17^{\circ} \mathrm{C}\right)$ for the entire $10 \mathrm{wk}$ period by utilising an automatically heated water circulatory system.

Fish $(\mathrm{n}=1200)$ were then transferred to 6 tanks, 200 fish and $250 \mathrm{l}$ of water in each. Three randomly selected tanks received a dose of 150 Diplostomum sp. cercariae fish $^{-1}$ (total of 30000 cercariae $\operatorname{tank}^{-1}$ ) for $30 \mathrm{~min}$. Cercariae were obtained from 25 naturally infected Lymnaea stagnalis snails (see Karvonen et al. 2003). To reach sufficient intensity of infection, the exposure was repeated after $3 \mathrm{~d}$. Three control tanks received sham exposure with water without parasites at both times. After the exposures, 300 control fish and 300 exposed fish were randomly assigned to 6 tanks ( $3+3$ tanks with control fish and exposed fish, respectively), 100 individuals and $1200 \mathrm{l}$ of water in each (remaining fish were used in other experiments). The experiment was then continued for $8 \mathrm{wk}$ to allow parasite development. Fish were fed daily with commercial fish food, and water temperature was kept constant at $17^{\circ} \mathrm{C}$ throughout the experiment. Fish groups in each tank were also weighed weekly for other purposes (mean fish weight at termination of the experiment was $41.9 \pm 0.9 \mathrm{~g}$ ). Afterwards, all fish were studied for parasites and lens diameter as described above.

As our purpose was to examine effects of the infection on lens size, we excluded the uninfected fish from the data (86 fish of the total of 1727 studied, 5.0\%) and refer to intensity of infection (number of parasites in infected fish; Bush et al. 1997) throughout the paper. We then calculated the difference in lens diameter and intensity of infection between the right and left eye for all individuals; positive values indicated a greater diameter or intensity of infection in the right lens and negative values a greater diameter or intensity in the left lens (Fig. 1). Individuals with no values from either 


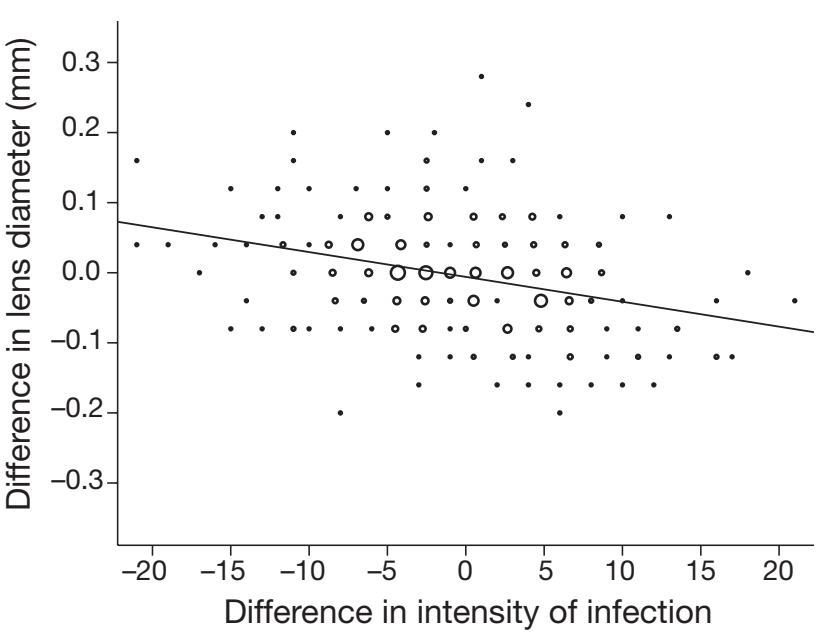

Fig. 1. Diplostomum sp. infecting Coregonus lavaretus. Relationship between difference in intensity of Diplostomum sp. infection and lens diameter between right and left lens of experimentally exposed whitefish $(\mathrm{n}=274)$. Positive values indicate higher values of the right lens and negative values those of the left lens. Size of each dot represents number of observations. Fitted line represents linear regression $\left(r^{2}=0.106\right)$

eye were excluded from the data. Relationships between difference in lens diameter and intensity of infection were analysed using linear regression, and, in the case of farmed salmon and sea trout, using stepwise linear regressions with fish population as the factor. In experimentally exposed whitefish, we also analysed the relationship between fish length and average lens diameter (mean for the right and left lens) using stepwise regression analysis with exposure status (exposed/control) as the factor. It should be noted that in the case of farmed and experimentally exposed fish, individuals from the same tank do not represent completely independent observations in a statistical sense. However, this is unlikely to affect the relationship between lens size and intensity of infection at the individual level. Furthermore, fish were taken from, or kept in, replicated tanks to exclude tank effects. Since the coverage of parasite-induced cataracts increases with intensity of infection (Karvonen et al. 2004a), and the position and coverage of the cataracts change in a 3-dimensional scale, the key determinant for the effects of infection in fish is the volume of the lens (see 'Discussion'). Thus, we also calculated the proportional difference in volume between the more and less heavily infected lens for each fish individual. However, lens diameter was used as a response variable in all regression analyses to meet the assumptions of normality of the data.

\section{RESULTS}

Intensity of infection in the 7 wild fish populations sampled from Bothnian Bay was highly variable, ranging from the mean of 2.8 (three-spined stickleback) to 83.8 (dace). The relationship between the difference in intensity of infection and difference in lens diameter of the right and left lenses was significant in 2 species,

Table 1. Mean intensity of Diplostomum sp. infection $( \pm \mathrm{SE})$ for the 7 fish species studied from Bothnian Bay, 4 species studied from a commercial fish farm, and experimentally exposed (E) and control (C) whitefish. Regression analyses give statistics for the relationship between difference in intensity of infection and lens diameter between right and left lens of individuals of each fish species (see Fig. 1). Reduction in lens volume indicates maximum proportional difference in lens volume within the fish individuals where the lens with a higher intensity of infection was smaller

\begin{tabular}{|c|c|c|c|c|c|c|}
\hline \multirow{2}{*}{$\begin{array}{l}\text { Population } \\
\text { Species }\end{array}$} & \multirow[t]{2}{*}{$\mathrm{n}$} & \multirow{2}{*}{$\begin{array}{l}\text { Intensity } \\
\text { of infection }\end{array}$} & \multicolumn{3}{|c|}{ Regression analysis } & \multirow{2}{*}{$\begin{array}{c}\text { Max. reduction } \\
\text { in lens volume }(\%)\end{array}$} \\
\hline & & & $\mathrm{r}^{2}$ & F & $\mathrm{p}$ & \\
\hline \multicolumn{7}{|l|}{ Bothnian Bay } \\
\hline Coregonus lavaretus & 72 & $8.6 \pm 1.0$ & 0.079 & 5.974 & 0.017 & 12.8 \\
\hline Osmerus eperlanus & 91 & $6.2 \pm 0.6$ & 0.083 & 8.060 & 0.006 & 18.1 \\
\hline Alburnus alburnus & 90 & $4.9 \pm 0.4$ & 0.006 & 0.569 & 0.453 & \\
\hline Leuciscus leuciscus & 96 & $83.8 \pm 5.8$ & 0.001 & 0.134 & 0.715 & \\
\hline Rutilus rutilus & 100 & $31.7 \pm 3.5$ & 0.002 & 0.230 & 0.633 & \\
\hline Gymnocephalus cernuus & 98 & $20.0 \pm 1.3$ & 0.004 & 0.367 & 0.546 & \\
\hline Gasterosteus aculeatus & 76 & $2.8 \pm 0.3$ & 0.010 & 0.785 & 0.379 & \\
\hline \multicolumn{7}{|l|}{ Fish farm } \\
\hline Salmo salar & 128 & $4.0 \pm 0.2$ & 0.0004 & 0.053 & 0.818 & \\
\hline S. trutta m. trutta & 151 & $3.8 \pm 0.2$ & 0.097 & 16.049 & 0.0001 & 16.5 \\
\hline S. trutta m. lacustris & 47 & $8.3 \pm 0.5$ & 0.066 & 3.192 & 0.081 & \\
\hline Coregonus lavaretus & 46 & $38.0 \pm 1.2$ & 0.275 & 16.693 & 0.0002 & 19.9 \\
\hline \multicolumn{7}{|l|}{ Experiment } \\
\hline Coregonus lavaretus C & 291 & $6.7 \pm 0.2$ & 0.103 & 33.310 & $<0.0001$ & 18.0 \\
\hline Coregonus lavaretus E & 274 & $46.5 \pm 0.7$ & 0.106 & 32.202 & $<0.0001$ & 19.2 \\
\hline
\end{tabular}




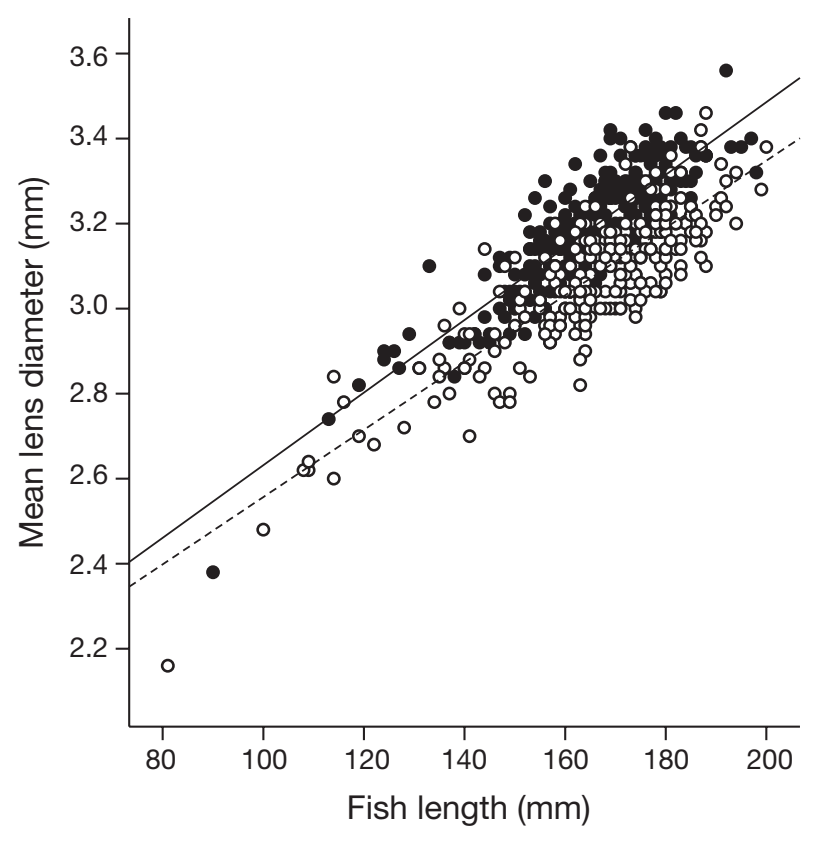

Fig. 2. Lens diameter in Coregonus lavaretus. Relationship between fish length and diameter of the lens (mean for right and left lens) in experimentally exposed whitefish $(0, n=$ 274) and controls $(\bullet, n=291)$. Fitted lines represent linear regression for exposed (--) and control (-) whitefish

whitefish and smelt, indicating that in individuals of these species the lens which harboured more parasites was smaller (Table 1). The highest proportional reduction in volume of the more heavily infected lens was $18.1 \%$ (smelt). A similar relationship was also observed in 2 species sampled from the fish farm, sea trout and whitefish (effect of fish population was not significant in either salmon or sea trout and was excluded from the final models). The highest proportional reduction in lens volume within an individual whitefish was $19.9 \%$ (Table 1).

In the experimental exposure, intensity of infection was significantly higher in the exposed whitefish compared to controls at the termination of the experiment (Mann-Whitney $U$-test: $Z=-20.576$, p < 0.001) (Table 1). The average lens diameter increased with fish size in both groups, but it was significantly smaller in the exposed fish (stepwise linear regression: $\mathrm{r}^{2}=0.765, F=917.181, \mathrm{p}<0.001$; coefficients: fish length: $t=39.431, \mathrm{p}<0.001$; exposure status: $t=$ -17.847, p < 0.001) (Fig. 2). Furthermore, at the individual level, increasing difference in intensity of infection in the right and left lenses resulted in increasing difference in lens diameter in both exposed and unexposed whitefish (Table 1, Fig. 1). Maximum proportional within-individual reduction in lens volume was $19.2 \%$.

\section{DISCUSSION}

The most notable effect of Diplostomum sp. infection in the eye lenses of fish is cataract formation, which can blind the fish (Shariff et al. 1980) and increase its susceptibility to predation (Seppälä et al. 2005). Infection is also known to impair feeding efficiency and growth of fish (Crowden \& Broom 1980, Owen et al. 1993, Buchmann \& Uldal 1994). In the present study, we observed that the infection also resulted in a smaller size of the eye lenses. Earlier, Shariff et al. (1980) reported smaller lens size in rainbow trout particularly in association with high-level chronic infections, but they did not quantify the changes in lens size. We observed an overall negative effect of the infection on lens size in fish subjected to experimental exposure. We also found that changes in lens size may take place even at low overall intensities of infection so that, for individual fish, the lens with a higher intensity of infection was smaller. This was observed both in wild and farmed fish populations, as well as in those exposed to experimental infection.

At least 2 mutually non-exclusive mechanisms can be responsible for the smaller lens size. First, to gain access to lenses, parasites must penetrate the lens capsule which may lead to leaking of the lens material to surrounding tissues and a subsequent reduction in lens size (see also Shariff et al. 1980). Second, parasites may interfere with the growth of the lens. After establishing themselves in the lens, metacercariae undergo extensive growth and development for a period of several weeks (Sweeting 1974, Seppälä et al. 2005), during which time they may affect lens physiology and exploit the energy otherwise used for lens growth. Since these mechanisms are indistinguishable using the present data, the term 'reduction in lens size' in this study refers to both of these mechanisms.

Interestingly, the relationship between asymmetry in intensity of infection and size of the right and left lens was not observed in all fish species; this was evident only in whitefish, smelt and sea trout. The reasons for this are unclear, but may include factors such as species-specific differences in physiology. It is also possible that differences in lens diameter between the eyes are affected by the overall intensity of infection, i.e. the effect may be observed either in low or high intensities of infection depending on the host species. For example, we observed no effect in the most heavily infected wild species, dace and roach, which is surprising given that the highest asymmetries in intensity of infection between the lenses were also recorded in these species. Reduction in lens size may reach an asymptote after a certain host species-specific level in intensity of infection when differences between more and less intensively infected lens are no longer observed. How- 
ever, this does not exclude the possibility that an overall reduction in lens size, similar to that in the experimentally exposed whitefish, has taken place.

Effects of the infection on lens size may also be affected by the pattern of parasite establishment. In the wild, metacercariae of Diplostomum sp. accumulate in fish over several years and intensity of infection increases with fish age (e.g. Burrough 1978, Marcogliese et al. 2001). Thus, it is likely that only a small number of parasites become established and develop in a lens at a given time. In this case, regardless of the mechanism underlying the reduction in lens size (see 'Discussion'), the effects of parasites on the lens may be relatively minor at each infection, but may accumulate over a long time period. At fish farms, on the other hand, exposure may resemble that of the experimental infection; lenses are subjected to a high number of new infections and subsequent parasite effects within a narrow time frame. However, if the effects of consecutive, low-level infections in nature were cumulative, i.e. no recovery of the lens occurs between these events, the significance of the pattern of establishment would become irrelevant. We could not analyse this from the present data on wild and experimentally exposed whitefish because of differences in the length distribution and intensity of infection in these fish. Ultimately, in order to separate the effects of different patterns of exposure, comparisons between experimentally exposed and totally uninfected fish would have to be made.

Reduction in lens size may have consequences for the severity of the deleterious effects of infection. Cataracts develop as a consequence of parasite metabolites and damage caused by parasite movements. Given that cataract coverage increases with intensity of infection (Karvonen et al. 2004a), reduced volume of the lens may intensify the input from each parasite individual, i.e. the same number of parasites may cause more severe cataracts in a smaller lens. In this study, the maximum reduction in volume of the more heavily infected lens of an individual fish was 12.8 to $19.9 \%$ depending on the fish species. This may be significant, for example, if it shifts individual hosts towards the point where the negative effects of the infection start to emerge (see Seppälä et al. 2005). In general, however, it would be difficult to separate the interrelated effects of intensity of infection and lens size on cataract coverage. The issue may be further complicated by changes in the location of the cataracts within the 3-dimensional lens, and when vision in one eye is impaired by infection, this may be compensated for by the other, less heavily infected eye. These aspects require substantial further investigation.

It is also possible that even a slight reduction in lens size may affect its overall optical performance. In fish, the aquatic environment neutralises the refractive power of the cornea and therefore most of this power is present in the lens (Evans 1998), which emphasises its key function in the vision of fish. The process of focusing of an image in vertebrates is also strongly associated with Matthiessen's ratio (the ratio between focal length and lens radius; Evans 1998), which may be sensitive to changes in the size of the lens. In such cases, vision could be affected by infection even at low intensities and in the absence of high cataract coverage, which is very important when considering the effects of infection.

Interestingly, within-individual effects were observed in whitefish in wild, farmed and experimentally exposed populations, emphasising the sensitivity of this species to parasite-induced changes in lens size. The result may be important from an applied point of view considering the growing importance of whitefish in aquaculture. Overall, possible changes in cataract coverage and optical performance of the lens following even slight parasite-induced changes in lens size are very important given the wide distribution of the parasite in natural waters and fish farms. Thus, further investigations are needed particularly on betweenspecies variation and the overall magnitude and significance of these changes in fish populations.

Acknowledgements. We thank H. Halonen for help in the laboratory, and T. Valtonen, E. T. Valtonen, P. Rintamäki and Y. Lankinen for help in providing the fish. Konnevesi Research Station provided facilities and technical assistance. This study was supported by the Academy of Finland Centre of Excellence in Evolutionary Research (A.K.) and a post-doctoral grant from the Academy of Finland (O.S.).

\section{LITERATURE CITED}

Buchmann K, Uldal A (1994) Effects of eyefluke infections on the growth of rainbow trout (Oncorhynchus mykiss) in a mariculture system. Bull Eur Assoc Fish Pathol 14:104-107

> Burrough RJ (1978) The population biology of two species of eyefluke, Diplostomum spathaceum and Tylodelphys clavata, in roach and rudd. J Fish Biol 13:19-32

Bush AO, Lafferty KD, Lotz JM, Shostak AW (1997) Parasitology meets ecology on its own terms: Margolis et al. revisited. J Parasitol 83:575-583.

- Crowden AE, Broom DM (1980) Effects of the eyefluke, Diplostomum spathaceum, on the behaviour of dace (Leuciscus leuciscus). Anim Behav 28:287-294

Evans DH (1998) The physiology of fishes. CRC Press, Boca Raton, FL

Field JS, Irwin SWB (1994) The epidemiology, treatment and control of diplostomiasis on a fish farm in Northern Ireland. In: Pike AW, Lewis JW (eds) Parasitic diseases of fish. Samara Publishing, Dyfed, p 87-100

Hudson PJ, Rizzoli A, Grenfell BT, Heesterbeek H, Dobson AP (2002) The ecology of wildlife diseases. Oxford University Press, Oxford

Karvonen A, Paukku S, Valtonen ET, Hudson PJ (2003) Transmission, infectivity and survival of Diplostomum 
spathaceum cercariae. Parasitology 127:217-224

Karvonen A, Seppälä O, Valtonen ET (2004a) Eye flukeinduced cataract formation in fish: quantitative analysis using an ophthalmological microscope. Parasitology 129: 473-478

Karvonen A, Seppälä O, Valtonen ET (2004b) Parasite resistance and avoidance behaviour in preventing eye fluke infections in fish. Parasitology 129:159-164

Karvonen A, Terho P, Seppälä O, Jokela J, Valtonen ET (2006a) Ecological divergence of closely related Diplostomum (Trematoda) parasites. Parasitology 133:229-235

Karvonen A, Savolainen M, Seppälä O, Valtonen ET (2006b) Dynamics of Diplostomum spathaceum infection in snail hosts at a fish farm. Parasitol Res 99:341-345

Marcogliese DJ, Dumont P, Gendron AD, Mailhot Y, Bergeron E, McLaughlin JD (2001) Spatial and temporal variation in abundance of Diplostomum spp. in walleye (Stizostedion vitreum) and white suckers (Catostomus commersoni) from the St. Lawrence River. Can J Zool 79:

Editorial responsibility: David Marcogliese, Montreal, Quebec, Canada
355-369

> Owen SF, Barber I, Hart PJB (1993) Low level infection by eye fluke, Diplostomum spp., affects the vision of three-spined sticklebacks, Gasterosteus aculeatus. J Fish Biol 42: 803-806

Seppälä O, Karvonen A, Valtonen ET (2005) Manipulation of fish host by eye flukes in relation to cataract formation and parasite infectivity. Anim Behav 70:889-894

Shariff M, Richards RH, Sommerville C (1980) The histopathology of acute and chronic infections of rainbow trout Salmo gairdneri Richardson with eye flukes, Diplostomum spp. J Fish Dis 3:455-465

Stables JN, Chappell LH (1986) The epidemiology of diplostomiasis in farmed rainbow trout from north-east Scotland. Parasitology 92:699-710

Sweeting RA (1974) Investigations into natural and experimental infections of freshwater fish by the common eyefluke Diplostomum spathaceum Rud. Parasitology 69: 291-300

Submitted: December 3, 2007; Accepted: March 11, 2008

Proofs received from author(s): May 24, 2008 